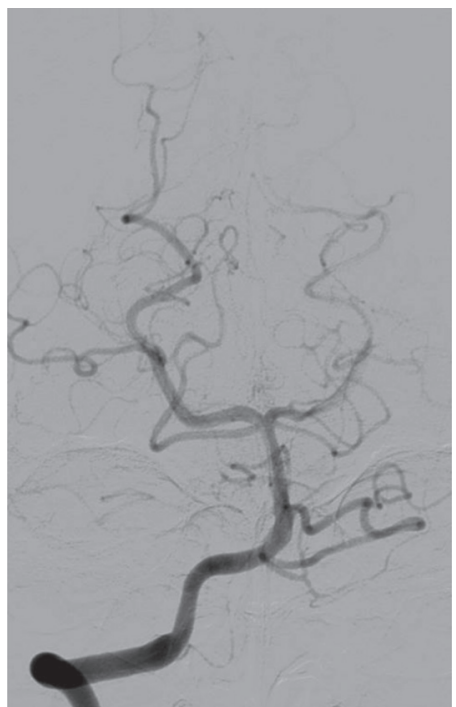

Abstract E-056 Figure 1

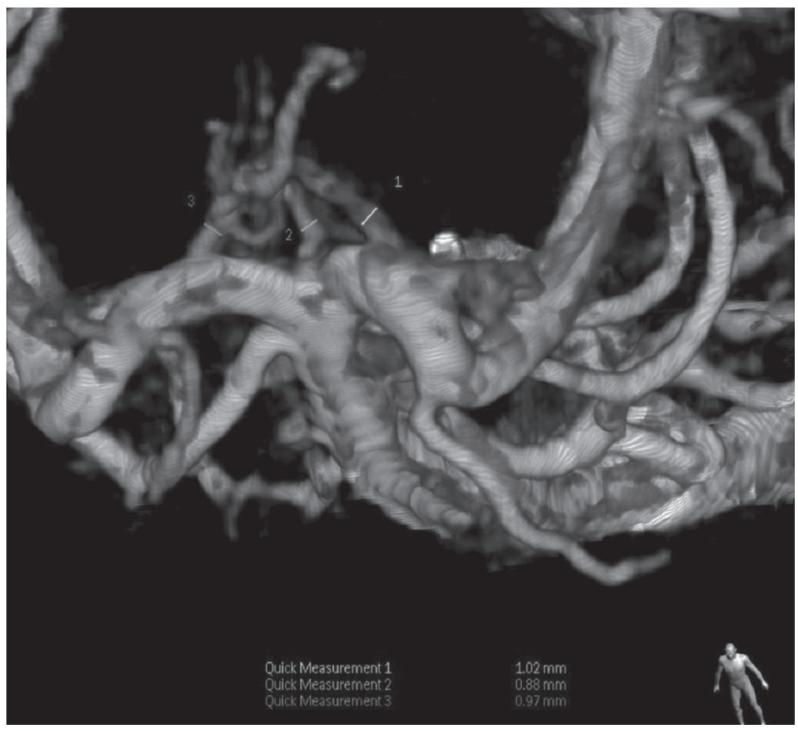

Abstract E-056 Figure 2

Disclosures B. Moon: None. S. Park: None. K. Jang: None. D. Jang: None.

\section{E-057 PROCESS IMPROVEMENT IN DOOR-TO-GROIN PUNCTURE TIMES AT A COMPREHENSIVE STROKE CENTER SHOWS A TREND TOWARDS REDUCED MORTALITY AT 90 DAYS}

${ }^{1} \mathrm{Y}$ Kayan, ${ }^{1} \mathrm{~J}$ Delgado Almandoz, ${ }^{2} \mathrm{M}$ Young, ${ }^{1} \mathrm{f}$ Fease, ${ }^{1} \mathrm{~J} S \mathrm{Scholz},{ }^{1} \mathrm{~A}$ Milner, ${ }^{2} \mathrm{~T}$ Hehr, ${ }^{3} \mathrm{M}$ Mulder, ${ }^{2} \mathrm{P}$ Roohani, ${ }^{2} \mathrm{R}$ Tarrel. ${ }^{1}$ Neurointerventional Radiology, Abbott Northwestern Hospital, Minneapolis, MN; ${ }^{2}$ Vascular Neurology, Abbott Northwestern Hospital, Minneapolis, MN; ${ }^{3}$ Critical Care, Abbott Northwestern Hospital, Minneapolis, MN

\subsection{6/neurintsurg-2016-012589.129}

Introduction Safe and effective endovascular treatment of acute ischemic stroke is dependent on prompt intervention. In light of the positive trials published last year, we intensified our efforts to improve the process of taking patients from the emergency department to the angiography suite for mechanical thrombectomy.

Methods At the beginning of 2015, a process improvement project to improve door-to-groin puncture times for mechanical thrombectomy for emergent large vessel occlusions was undertaken at our institution. After a systematic analysis of the process, the following key changes were standardized: early consultation with the neurointerventionalist for patients presenting with a high National Institutes of Health Stroke Scale (NIHSS $\geq 6$ ), the stroke neurologist and the neurointerventionalist meet the patient in the emergency department for transfers or meet in the CT department for non-transfers, elimination of emergency physician triage for medically stable patients, elimination of lab tests such as creatinine, establishment of a minimum level of clinical information required for the sedation nurse to care for stroke patients safely, institution of a "stroke bag" containing all necessary devices to perform efficient thrombectomy, standardized setup of the procedure table and devices for all operators, calling for patients when the sedation nurse is ready without waiting for the entire team, elimination of unnecessary preoperative steps (e.g. Foley catheter placement, groin shaving, checking of distal pulses), and institution of an abbreviated time-out procedure. All attempted thrombectomies performed in 2013 and 2014 were compared to those performed in 2015 visà-vis the following variables: door-to-groin puncture time, symptomatic intracranial hemorrhage $(\mathrm{SICH})$ rate as defined by SITS-MOST criteria, 90 day mortality rate, and 90 day rate of good clinical outcome defined by a modified Rankin Scale (mRS) of 0-2. A one-tailed Student's t-test and Fisher's exact tests were performed. A p-value $<0.05$ was considered statistically significant.

Results From January 2013 to December 2015, we attempted 108 mechanical thrombectomies. Of these, 43 were in 2015 (40\%). Both the mean and median door-to-groin puncture times were significantly reduced from the previous two years (35 minutes versus 89 minutes and 22 minutes versus 73 minutes, respectively, $\mathrm{p}<0.001)$. There was a trend towards reduced mortality at 90 days $(16 \%$ versus $26 \%, \mathrm{p}=0.166)$. The rates of SICH and mRS 0-2 at 90 days were not significantly different (see Table 1).

Conclusion Our systematic process improvement initiative significantly reduced door-to-groin puncture times and showed a trend towards reduced mortality at 90 days. A multidisciplinary approach and ensuring hospital system investment are key to an effective process.

\begin{tabular}{llll}
\multicolumn{4}{l}{ Abstract E-057 Table 1} \\
\hline & 2013 and 2014 & 2015 & p-value \\
\hline Thrombectomies & 65 & 43 & \\
Door-to-groin puncture & 89 & 35 & $<0.001$ \\
Mean time (minutes) & 73 & 22 & \\
Median time (minutes) & & & \\
SICH & $3(5 \%)$ & $3(7 \%)$ & 0.452 \\
90 day mortality & $17(26 \%)$ & $7(16 \%)$ & 0.166 \\
90 day mRS 0-2 & $24(37 \%)$ & $18(42 \%)$ & 0.376 \\
\hline
\end{tabular}

Disclosures Y. Kayan: 2; C; Medtronic, Penumbra. J. Delgado Almandoz: 2; C; Medtronic, Penumbra. M. Young: None. J. Fease: None. J. Scholz: None. A. Milner: None. T. Hehr: None. M. Mulder: None. P. Roohani: None. R. Tarrel: None. 